\title{
PREVALENCIA DE ENDOMETRITIS CLÍNICA Y SUBCLÍNICA EN VAQUILLAS REPETIDORAS MANTENIDAS EN AMBIENTE TROPICAL HUMEDO
}

\author{
Prevalence of bovine clinical and subclinical endometritis in repeat breeder heifers \\ under humid tropical environment
}

\author{
M.A. Gutierrez-Reinoso', J.J. Masaquiza-Aragon ${ }^{1,2}$, R. Quinteros-Pozo², M. Garcia-Herreros ${ }^{1,3}$ \\ http://dx.doi.org/10.18548/aspe/0002.22
}

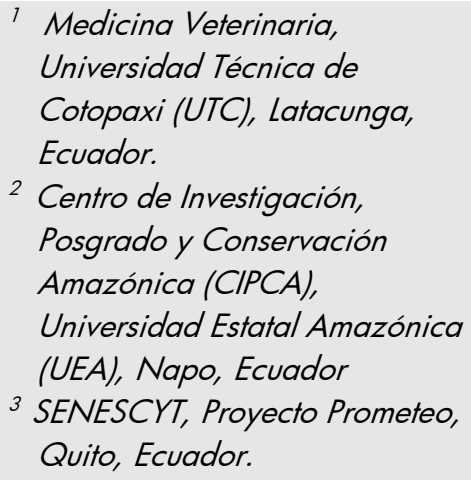

Medicina Veterinaria, Universidad Técnica de Cotopaxi (UTC), Latacunga, Ecuador.

2 Centro de Investigación, Posgrado y Conservación Amazónica (CIPCA), Universidad Estatal Amazónica (UEA), Napo, Ecuador

3 SENESCYT, Proyecto Prometeo, Quito, Ecuador.

E-mail: herrerosgm@gmail.com

\section{RESUMEN}

El objetivo del presente estudio fue evaluar la prevalencia de endometritis mediante una combinación de análisis citológico y microbiológico en muestras endometriales de novillas repetidoras (Bos taurus) mantenidas en ambiente tropical húmedo con el fin de aplicar un tratamiento diferenciado que optimice la fertilidad bajo dicho ambiente. Se realizó IATF en vaquillas Brown Swiss y los animales no gestantes (repetidores; $n=14$ ) se sometieron a un análisis citológico mediante la técnica Cytobrush (CB) pre y pos-tratamiento diferenciado. Se identificaron 9 especies de microorganismos que disminuyeron a 7 en las muestras pos-tratamiento. La determinación del tipo de endometritis se realizó en frotis mediante el índice de neutrófilos polimorfonucleares ( $\%$ PMN). La prevalencia pre-tratamiento de endometritis fue del $36,8 \%$, del cual el $28,6 \%$ fue subclínica y el $71,4 \%$ clínica. La prevalencia pos-tratamiento supuso un total del $50 \%$ positivo a endometritis, del cual el 71,4\% fue subclínica y el $28,6 \%$ clínica. La tasa de fertilidad pos-tratamiento incremento un $50 \%$. La prevalencia del tipo de endometritis diagnosticada tras el tratamiento invirtió los porcentajes entre endometritis clínica y subclínica, lo cual influyó en la severidad de dicha patología y en el índice de fertilidad. La detección y clasificación del tipo de endometritis es fundamental para elegir el tratamiento diferenciado más eficiente en novillas repetidoras (Bos taurus) en ambientes tropicales.

Palabras clave: Citología, microbiología, endometritis, bovino. 
The aim of this study was to evaluate the prevalence of endometritis through a combination of cytological and microbiological analysis in endometrial samples from repeater heifers (Bos taurus) mantained under humid tropical environment in order to apply a specific treatment to optimize fertility under this environment. Brown Swiss heifers were used (repeat breeder heifers; $\mathrm{n}=14$ ) to perform a cytological analysis by using Cytobrush (CB) technique before and after a differentiated treatment. In pre-treatment samples, 9 species of microorganisms were identified, however, this number decreased to 7 in post-treatment samples. Endometritis determination (clinical or subclinical) was performed by using smears (index of polymorphonuclear neutrophils (PMN\%)). The prevalence of endometritis during pre-treatment was $36.8 \%$ (overall), of which $28.6 \%$ and $71.4 \%$ corresponded to subclinical and clinical endometritis respectively. The prevalence of endometritis post-treatment was 50\% (overall), of which $71.4 \%$ and $28.6 \%$ corresponded to subclinical and clinical endometritis, respectively. The fertility rate posttreatment increased a 50\%. The combination of cytological and microbiological analysis allowed an efficient diagnosis in the detection and classification of the type of endometritis in order to select the most efficient treatment. The prevalence rate diagnosed involved a significant change after treatment application, reversing the percentages between clinical and subclinical endometritis, which would indicate a positive effect of differential treatment on the number of positive cases, the severity of this disease and fertility rate in tropical environments.

Keywords: Cytology, microbiology, endometritis, bovine

\section{INTRODUCCION.}

Los problemas reproductivos son frecuentes en bovinos mantenidos en ambientes tropicales y están condicionados por diferentes factores. Las condiciones ambientales tropicales (humedad y temperatura), el manejo, la falta de energía en la dieta, un deficiente control sanitario, el tipo de geografía irregular y los genotipos utilizados dificultan frecuentemente alcanzar niveles eficientes en las tasas de fertilidad. Por ello, se han desarrollado estudios respecto a protocolos de inseminación artificial a tiempo fijo (IATF), con el fin de mejorar los parámetros reproductivos. Sin embargo, se han obviado características fundamentales, como es el estado funcional del endometrio, a excepción de aquellos animales que manifiestan alguna sintomatología clínica evidente. A pesar de que el endometrio bovino posee numerosos mecanismos de defensa (células epiteliales pseudoestratificadas, moco secretado por las glándulas endometriales y la acción de las células polimorfonucleares y anticuerpos humorales), que lo protege de agentes invasivos inespecíficos (Dhaliwal, 2001) no queda exento de poder ser invadido y perjudicado por diferentes microorganismos que pueden desencadenar infecciones subclínicas. En estudios recientes (Green et al., 2011) se observó que la endometritis subclínica (ES) modifica las concentraciones de esteroides ováricos afectando la calidad del ovocito, y esto podría explicar en parte las tasas de concepción más bajas y el mayor intervalo entre el parto y la concepción, que se asocian a menudo con otras causas, tales como la endometritis de tipo subclínico. Aunque frecuentemente pase desapercibido, en este tipo de patologías dan lugar a fenómenos de modificación del epitelio uterino, lo cual repercute negativamente en los índices de concepción, y como consecuencia, en la productividad del animal. Las endometritis se clasifican de forma general en dos tipos; la clínica (caracterizada por desarrollar descargas purulentas desde el útero al exterior incluso pasadas 3-4 semanas postparto) y la subclínica (caracterizada por la presencia de neutrófilos con ausencia de descarga, pero con consecuencias severas en la fertilidad) (Sheldon et al., 2008).

Las endometritis subclínicas discurren con una inflamación superficial del endometrio que apenas alcanza el estrato esponjoso aunque en ocasiones, puede haber pérdida de la superficie epitelial, aparición subepitelial de células inflamatorias, congestión vascular y edema acompañado por la aparición de linfocitos en la lámina propia. Los animales afectados pasan desapercibidos debido a la ausencia de signos clínicos. En cuanto a agentes etiológicos, Actinomyces pyogenes aparece frecuentemente en los diagnósticos microbiológicos, el cual puede estar acompañado por otros microorganismos patógenos concomitantes tales como Archanobactetiun pyogenes, Escherichia coli, Fusobacterium necrphorum y Bacteroides spp, contribuyendo a la producción de toxinas, que como consecuencia elevan a niveles no fisiológicos las concentraciones de PGF2-alpha, lo que produce un retraso de la ciclicidad del animal (Seals et al., 2002). Debido a lo expuesto, el objetivo del presente estudio fue determinar la prevalencia de endometritis mediante análisis citológico y microbiológico de muestras endometriales tomadas en novillas repetidoras (Bos taurus) en ambiente tropical.

\section{MATERIAL Y METODOS}

Se tomaron 38 vaquillas de la raza Brown Swiss mantenidas a libre pastoreo con buenas condiciones sanitarias y de manejo a las que se les aplicó un protocolo de sincronización para realizar inseminación artificial a tiempo fijo (IATF). El diseño experimental fue llevado a cabo en el Centro de Investigación, Posgrado y Conservación Amazónica 
(CIPCA) perteneciente a la Universidad Estatal Amazónica (UEA) en el cantón Tena, provincia del Napo, Ecuador (latitud: S $1^{\circ} 10^{\prime} / \mathrm{S} 1^{\circ} 0^{\prime}$; longitud: W $77^{\circ} 45^{\prime} / \mathrm{W} 77^{\circ} 30^{\prime}$; altitud: $\left.510 \mathrm{msnm}\right)$. El protocolo consistió en colocar un implante Crestar $\AA$ (Intervet International B.V. Boxmeer - Holanda), compuesto por $3 \mathrm{mg}$ de Norgesomet (análogo sintético de P4), $5 \mathrm{mg}$ de Valerato de Estradiol (intramuscular) y 400 UI eCG (Folligon) el día 0. La retirada del implante se realizó el día 7 y se aplicaron 150 ug de PG2a (Bioprost D biotay) + 1 mg ECP (Cipionato de Estradiol-Pfizer). Finalmente, el dia 9 se realizó la IATF simultáneamente a la aplicación de 0,2 mg de GnRH. Tras la sincronización e inseminación se realizó un diagnóstico de gestación mediante ultrasonografía (Ibex Pro, transductor lineal de $5.0 \mathrm{MHz}$ ) a los 45 días pos-IA. Los individuos diagnosticados no gestantes (repetidores; $\mathrm{n}=14$ ) se sometieron a un análisis citológico para la posterior determinación de la microbiota endometrial mediante cultivo microbiológico y antibiograma (Animalab Cia. Ltda). El análisis citológico se llevó a cabo mediante la técnica de citología endometrial con cepillos endocervicales (Cytobrush, CB), se trata de una técnica rápida, sensible, eficaz y económica y consiste en un dispositivo dotado de un cepillo para tomar muestras a nivel de endometrio, para posteriormente realizar un frotis que será teñido con Tinción 15 (Biopur) (Kasimanickam et al., 2005). Para la obtención de las muestras, se utilizan pistoletas de acero inoxidable, a las que se le adosan en su extremo anterior cepillos estériles comúnmente usados en ginecología humana. Todo esto es protegido mediante una vaina sanitaria plástica, para evitar la contaminación del cepillo con células del cuello y del vestíbulo. Para la obtención de las muestras, se utilizan pistoletas de acero inoxidable, a las que se le adosan en su extremo anterior cepillos estériles comúnmente usados en ginecología humana. Todo esto es protegido mediante una vaina sanitaria plástica para evitar la contaminación del cepillo con células del cuello y del vestíbulo vaginal. En esta técnica se toma como referencia el índice de neutrófilos polimorfonucleares (\% PMN) en el frotis para diagnosticar la existencia de procesos inflamatorios y clasificar el tipo de endometritis (clínica o subclínica). La endometritis subclínica se caracteriza por la presencia de $<5 \%$ células PMN (particularmente neutrófilos) y por tanto en el presente estudio, la evaluación de 200 células por frotis (epiteliales e inflamatorias) determinó la clasificación de individuos positivos y negativos a dicha patología (Rinaudo et al., 2012). Posteriormente, dichos individuos se sometieron a un tratamiento diferenciado (antibiótico + antiinflamatorio (Flunixin meglumine) de acuerdo al antibiograma (ver Tabla 1) y por último se realizaron nuevamente análisis citológicos y microbiológicos posttratamiento. El análisis de los datos se llevó a cabo mediante la utilización del software estadístico SPSS v. 15 para Windows. Debido a que la exploración de los datos determino una distribución no normal de éstos, se procedió a la aplicación del test chi-cuadrado para la comparación de las diferentes variables. Las diferencias se consideraron significativas cuando $\mathrm{P}<0,05$.

Tabla 1. Microorganismos obtenidos a partir del cultivo en la primera y segunda toma de muestras mediante el uso de Cytobrush (CB) y su correspondiente tratamiento antibiótico

\begin{tabular}{llccc}
\hline$N^{\circ}$ Microorganismo aislado & $\begin{array}{c}\mathrm{N}^{\circ} \text { anim. Infectados } \\
\text { Antes del tratamiento }\end{array}$ & $\begin{array}{c}\mathrm{N}^{\circ} \text { anim. Infectados } \\
\text { Post tratamiento }\end{array}$ & $\begin{array}{c}\text { Tratamiento } \\
\text { Aplicado }\end{array}$ \\
\hline 1 & Staphylococcus aureaus & 6 & 3 & Ceftiofur/Eritromic. \\
2 & Enterococcus faealis & 1 & 0 & Amoxicil.+Ac. Clav. \\
3 & Streptococcus ( -hemolít.) & 7 & 3 & Amoxicil.+Ac. Clav. \\
4 & Candida albicans & 5 & 0 & Ceftiof./Amox./Clav. \\
5 & Escherichia Coli & 1 & 0 & Eritromicina \\
6 & Bacillus spp & 4 & 1 & Eritromicina \\
7 & Coliformes & 2 & 1 & Ceftiofur/Eritromic. \\
8 & Streptococcus agalactiae (B-hemolít.) & 3 & 1 & Ceftiofur/Eritromic. \\
9 & Streptococcus viridans & 1 & 0 & Amoxicil.+Ac. Clav. \\
10 & Staphylococcus epidermidis & 0 & 2 & Ceftiofur \\
11 & Acynetobacter spp & 0 & 1 & Amoxicil.+Ac. Clav. \\
\hline
\end{tabular}




\section{RESULTADOS}

En la primera muestra tomada pre-tratamiento, se identificaron 9 especies de microorganismos de diferentes géneros (Streptococcus, Staphylococcus, Cándida, Bacillus, Enterococcus y Escherichia) que disminuyeron a 7 especies de diferentes géneros (Streptococcus, Staphylococcus, Bacillus, Escherichia y Acynetobacter) en la segunda muestra tomada postratamiento.

La prevalencia pre-tratamiento de endometritis fue del $36,8 \%$ (14/38), del cual el 28,6\% (4/14) correspondió a un cuadro de endometritis subclínica y el $71,4 \%(10 / 14)$ a endometritis clínica. La prevalencia pos-tratamiento supuso un total del $50 \%$ $(7 / 14)$ positivo a endometritis, del cual el $71,4 \%$
(10/14) correspondieron a endometritis subclínica y el $28,6 \%(4 / 14)$ a endometritis clínica. Sin embargo, según el valor de Chi-cuadrado calculado, la tendencia observada demuestra no mantener diferencias estadísticamente significativas $(P>0,05)$ con una tendencia lineal esperada (proporción de animales infértiles de acuerdo a \%PMN [N $\mathrm{N}^{\circ} \mathrm{Cel}$. PMN/N ${ }^{\circ} \mathrm{Cel}$. Epitel.]), lo cual indicaría que la infertilidad en novillas tras la $2^{a}$ IA no varía en función del \%PMN n/CE, es decir, la prevalencia de endometritis ya sea de tipo clínico o subclínico no determina un aumento o una disminución de las tasas de fertilidad, pues las novillas no quedan gestantes sea cual sea el tipo de endometritis diagnosticada. La tasa de fertilidad postratamiento $\left(2^{a}\right.$ IA) se incrementó a un $50 \%(7 / 14$ novillas gestantes).

Tabla 2. Porcentaje de Células Polimorfonucleares (PMNn) y Células Epiteliales (CE), en las dos tomas de muestras mediante Cytobrush (CB)

\begin{tabular}{|c|c|c|c|c|c|c|}
\hline \multirow[b]{2}{*}{$\mathrm{N}^{\circ}$} & \multicolumn{3}{|c|}{$1^{a}$ Toma CB } & \multicolumn{3}{|c|}{$2^{a}$ Toma CB } \\
\hline & $\begin{array}{l}\text { N CÉL.PMN } \\
\text { (neutrifilos) }\end{array}$ & N CÉL EPIT. & $\begin{array}{c}\text { Relación } \\
\text { PMN/EP (\%) }\end{array}$ & $\begin{array}{l}\text { CÉL. PMN } \\
\text { (neutrófilos) }\end{array}$ & $\begin{array}{c}\mathrm{N}^{\circ} \mathrm{CEL} . \\
\text { EPIT. }\end{array}$ & $\begin{array}{c}\text { Relación } \\
\text { PMN/EP (\%) }\end{array}$ \\
\hline 1 & 12 & 188 & $6 \%$ & - & - & - \\
\hline 2 & 8 & 192 & $4 \%$ & 3 & 197 & $2 \%$ \\
\hline 3 & 38 & 162 & $23 \%$ & 11 & 189 & $6 \%$ \\
\hline 4 & 10 & 190 & $5 \%$ & 6 & 194 & $3 \%$ \\
\hline 5 & 5 & 195 & $3 \%$ & - & - & - \\
\hline 6 & 14 & 186 & $8 \%$ & 2 & 198 & $1 \%$ \\
\hline 7 & 17 & 183 & $9 \%$ & - & - & - \\
\hline 8 & 21 & 179 & $12 \%$ & 8 & 192 & $4 \%$ \\
\hline 9 & 19 & 181 & $10 \%$ & 10 & 190 & $5 \%$ \\
\hline 10 & 15 & 185 & $8 \%$ & - & - & - \\
\hline 11 & 23 & 177 & $13 \%$ & 7 & 193 & $4 \%$ \\
\hline 12 & 32 & 168 & $19 \%$ & - & - & - \\
\hline 13 & 6 & 194 & $3 \%$ & - & - & - \\
\hline 14 & 6 & 194 & $3 \%$ & - & - & - \\
\hline
\end{tabular}

\section{DISCUSIÓN}

A pesar de que los protocolos de IATF han mejorado sustancialmente los niveles reproductivos obtenidos en ambientes tropicales con tasas de gestación entorno al 54\% (López, et al., 2014), existe la necesidad de controlar otros factores que mejorarían notablemente dichos porcentajes, como es el caso del control sanitario y el diagnóstico de patologías carentes de sintomatología como es el caso de la endometritis subclínica. La endometritis subclínica se encuentra entre las causas responsables del fracaso en la gestación y la repetición de celos en el ganado bovino, siendo difícil su diagnóstico ya que los signos clínicos de esta patología suelen pasar desapercibidos debido a que el estudio bacteriológico del mucus uterino no refleja el estado del endometrio (Dhaliwal et al., 2001). Sin embargo, tal y como se demuestra en el presente estudio, la combinación del análisis citológico endometrial con un análisis microbiológico cuidadoso del útero, pueden contribuir a realizar un diagnóstico mucho más eficiente. En el presente estudio, la prevalencia de endometritis fue del $36,8 \%$, del cual el $28,6 \%$ corresponde a endometritis subclínica y el $71,4 \%$ a endometritis clínica a partir de la primera toma de muestras mediante $C B$, mientras que en la segunda toma, tras aplicar el tratamiento correspondiente, disminuyó al 50\%, del cual el 71,4\% correspondió a endometritis subclínica y el $28,6 \%$ a endometritis clínica. Estos resultados resaltan la importancia de la 
aplicación del tratamiento adecuado, debido a que un estudio llevado a cabo por Maurino, et al. (2012) se reportó que el porcentaje de endometritis subclínica fue del $44,4 \%$ remarcando la necesidad de aplicar un tratamiento efectivo para estos casos. Respecto al diagnóstico microbiológico en animales sanos, Escherichia coli fue el microorganismo de mayor incidencia en muestras tomadas de cérvix y útero $(28,1$ y $23,1 \%$ respectivamente) y con menor frecuencia se aislaron otras enterobacterias y bacterias del género Staphylococcus y Streptococcus (Fernandez et al., 1984). Sin embargo, en el presente estudio, los resultados obtenidos en el primer cultivo comparado con el segundo revelan que hay una tendencia descendente de la presencia de Streptococcus gamma hemolíticos, Staphylococcus aureaus, Cándida albicans, Bacillus spp, Streptococcus agalactiae (beta hemolíticos), Coliformes, Enterococcus faealis, Escherichia Coli y Streptococcus viridans. Sin embargo, Fernandez-Martínez et al. (2006), reportaron que en el endometrio de animales clínicamente sanos los microorganismos residentes actúan como un factor de defensa primaria del huésped siendo probable que pueda protegerlo de la invasión de otros microorganismos patógenos por mecanismos de competición. Esto reforzaría la teoría de la necesidad de la existencia de una flora bacteriana saprofita para evitar desequilibrios de la microbiota que den lugar a la proliferación de microorganismos que normalmente no son patógenos, pero que a partir de situaciones de disbiosis, podrían llegar a serlo.

\section{CONCLUSIONES}

La prevalencia del tipo de endometritis diagnosticada sufrió un cambio significativo tras la aplicación del tratamiento invirtiendo los porcentajes entre endometritis clínica y subclínica, lo cual indicaría un efecto positivo respecto al número de casos endometritis, la severidad de dicha patología y el índice de fertilidad. La detección y clasificación del tipo de endometritis es fundamental para elegir el tratamiento diferenciado más eficiente en novillas repetidoras (Bos taurus) en ambientes tropicales.

\section{REFERENCIAS}

- Dhaliwal GS, Murray RD, Woldehiwet Z. Some aspects of immunology of the bovine uterus related to treatments for endometritis. Anim Reprod Sci. 2001; 67:135-52.

- Fernández A, Dimoso ZJK. Estudio cualitativo y cuantitativo de la flora bacteriológica de las secreciones cervicouterinas de vacas clínicamente sanas. Facultad de Ciencia Animal. Universidad Central "Marta Abreu" de Las Villas. Santa Clara. Cuba. 1984

- Fernández-Martínez A., Silveira-Prado EA, LópezRojas OF. Uterine infections in bovine female
REDVET Vol. VII, $N^{\circ}$ 10, 2006. http://www.veterinaria.org/revistas/redvet/n 101 006.html

- Green MP, Ledgard AM, Beaumont SE, Berg MC, McNatty KP, Peterson AJ, Back PJ. Long-term alteration of follicular steroid concentrations in relation to subclinical endometritis in postpartum dairy cows. J Anim Sci. 2011; 89:3551-3560.

- Kasimanickam R, Duffield TF, Foster RA, Gartley CJ, Leslie KE, Walton JS, Johnson WH. A comparison of the cytobrush and uterine lavage techniques to evaluate endometrial cytology in clinically normal postpartum dairy cows. Can Vet J. 2005; 46:255259.

- Lopez LM, Grey TW, Chen M, Hiller JE. Strategies for improving postpartum contraceptive use: evidence from non-randomized studies. Cochrane Database Syst Rev. 2014; 11:CD011298.

- Maurino A, Bernardi S, Rinaudo A, Marini PR. Prevalence of subclinical endometritis before and four hours after artificial insemination in heifers Spermova 2012; 2: 47-48.

- Seals RC, Matamoros I, Lewis GS. Relationship between postpartum changes in 13, 14-dihydro-15keto PGF2alpha concentrations in Holstein cows and their susceptibility to endometritis. J Anim Sci. 2002; 80:1068-1073.

- Sheldon IM, Williams EJ, Miller AN, Nash DM, Herath S. Uterine diseases in cattle after parturition. Vet J. 2008; 176:115-121.

- Rinaudo A, Bernardi SF, Marini PR. Punto de corte del porcentaje de neutrófilos para el diagnóstico de endometritis subclínica en vacas lecheras XIII Jornadas de Divulgación Técnico Científicas 2012 Facultad de Ciencias Veterinarias (UNR), Argentina 2012. 